\title{
INVESTIGATION OF FLOW IN DATA RACK
}

\author{
Lukáš MANOCH, Jiří NOŽIČKA, Petr POHAN
}

\begin{abstract}
The main purpose of this paper was to set up a functioning numerical model of data rack verified by an experimental measurement. The verification of the numerical model was carried out by means of the PIV method (Particle Image Velocimetry). The numerical model was "found" while using the assumed and preset values from the experimental measurement which represent boundary conditions. The server model was conceived as a four-channel with a controlled flow rate without simulation of heat transfer. The flow rate in each channel was implemented by means of pressure loss. The numerical model was further used for simulation of several phases and configurations of data rack (21U rack space) fitted with two server workstations Dell Precision R5400. The flow field in the inlet of data rack in the front of the workstations were observed and evaluated in such a way that a $2 U$-dimensional free space between the workstations was being left and the remaining inlet space was blanked-off/fully opened. The results of this paper will serve for designing optimization treatment of data rack from the viewpoint of cooling efficiency both within the data rack and within the data center design.
\end{abstract}

\section{Introduction}

The purpose of this paper was to set up a functioning numerical model, verified by an experimental measurement, of data rack fitted with two rack workstations Dell Precision R5400 (Figure 1), 2 U rack space each. The workstations were set in the middle part of the cabinet (Figure 2) and a $2 \mathrm{U}$-dimensional free space between the workstations was being left.

\footnotetext{
Lukáš Manoch, Czech Technical University in Prague, Faculty of Mechanical Engineering, Technická 4, Praha 6, 166 07, Czech Republic, lukas.manoch@fs.cvut.cz.

Jiři Nožička, Czech Technical University in Prague, Faculty of Mechanical Engineering, Technická 4, Praha 6, 166 07, Czech Republic, jiri.nozicka@fs.cvut.cz.

Petr Pohan, CONTEG, spol. s.r.o., Na Vítězné pláni 1719/4, Praha 4, 140 00, Czech Republic, p.pohan@conteg.cz.
}

This is an Open Access article distributed under the terms of the Creative Commons Attribution License 2.0, which permits unrestricted use, distribution, and reproduction in any medium, provided the original work is properly cited. 


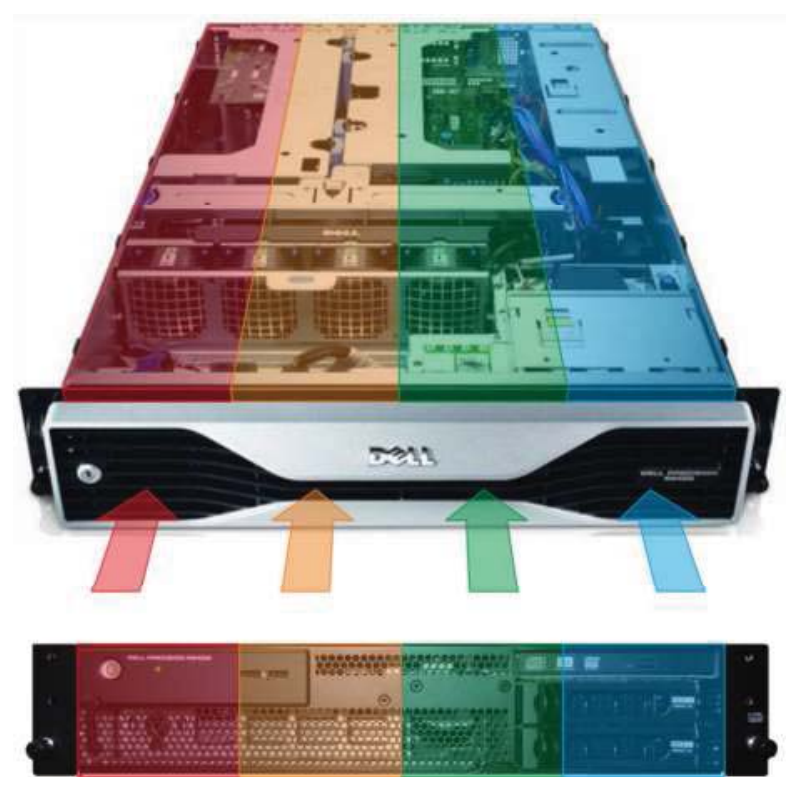

Figure 1: Dell Precision R5400

Two suitable compilations of cabinets were being considered for the work purpose. As for the first compilation, the inlet space was fully opened. In case of the other compilation, the inlet space was fully blanked off and the medium was flowing only through the rack workstations (Figure 2).
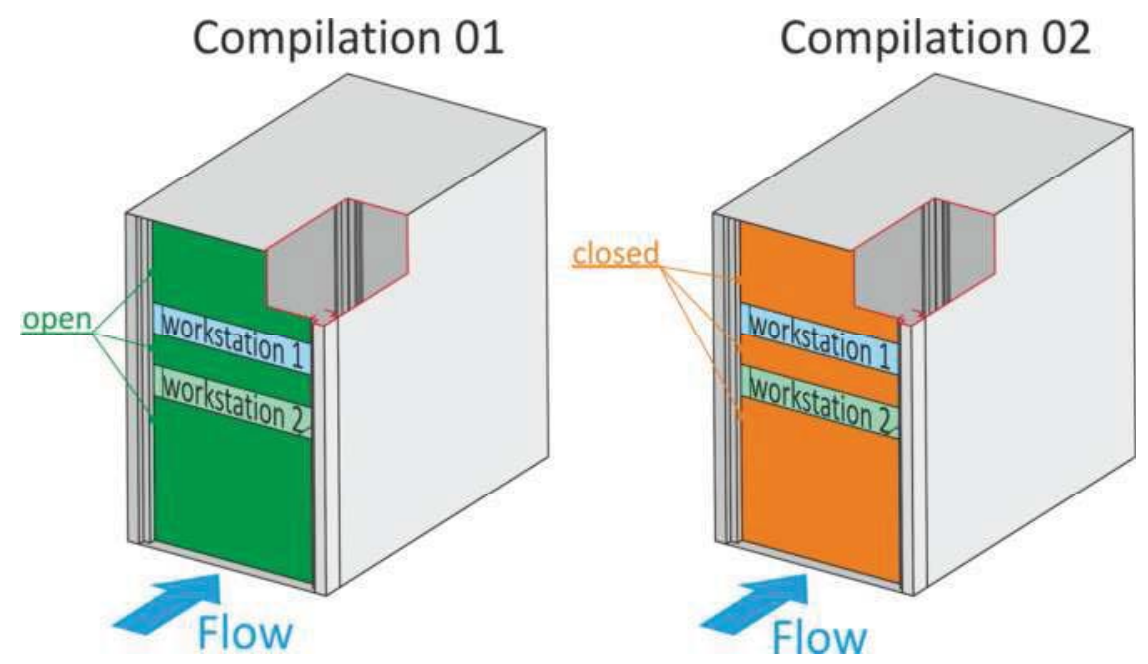

Figure 2: Compilation of cabinets

\section{Experiment}

The experimental part consisted of determining the shape of velocity field and defining the temperature in the inlet of the rack workstation Dell Precision R5400. The velocity and temperature field was measured in the $1-\mathrm{cm}$ distance from the front of the workstation without the front case panel (Figure 3) because of the substantial geometric complexity, which could considerably complicate the identification of the flow 
parameters. In the cross-sectional dimension of the workstation, several planes with $1 \mathrm{U}$ rack space $(1$ rack unit $=1 \mathrm{U}=4.445 \mathrm{~cm}$ ) were determined, where the measurement of the velocity fields was carried out. The velocity fields consequently constituted the general velocity field in the inlet of the rack workstation.

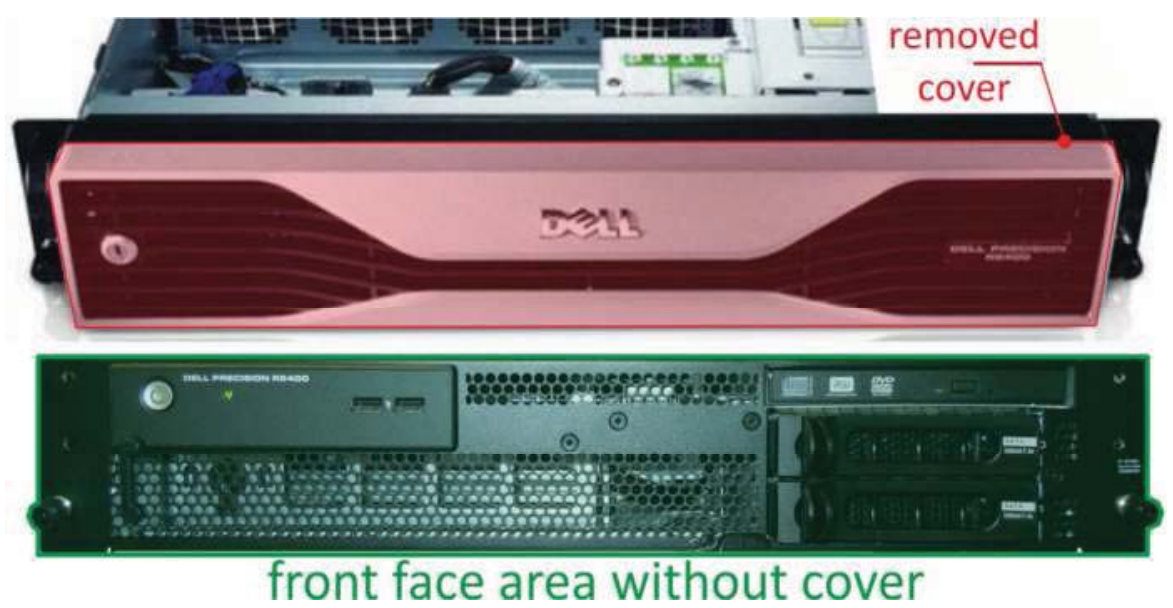

Figure 3: Workstation with and without front panel

The measurement was carried out by the PIV method (Particle Image Velocimetry). A construction, with camera fixed firmly against the laser on a moving support, was set. Thus the same position and angular location of the camera against the laser during the whole measurement was achieved. This compilation was set up so that the laser sheet was perpendicular in a maximum way to the front of the workstation. The moving support consequently moved in the cross-sectional direction of the workstation in a regime mentioned above.

A camera flowSense 4M (12 bites, 4 megapixels) together with a pulse laser Nd:YAG NewWave Gemini $120 \mathrm{~mJ}$ in pulse was used. Dynamic Studio program was used for synchronization and evaluation. For calculating vectors during evaluation, a method of multi step correlation with initial size IA (Interrogation area) 64×64 pixels and resultant size $32 \times 32$ pixels was used. A filter peak ratio, where only vectors with ratio better than 1.1 were accepted within the filter, was used for data validation. All results are timeaveraged from 100 measurements.

It was assembled structure (Figure 4) in which the position of the camera to the laser was firmly fixed on a movable slide. This was achieved by the same positional and angular position of the camera to the laser during the whole measurement. This assembly was then adjusted so that the laser sheet was as much as possible perpendicular to the front face of the workstation. Movable slide is then moved in the transverse direction of the workstations in the above-defined mode. 


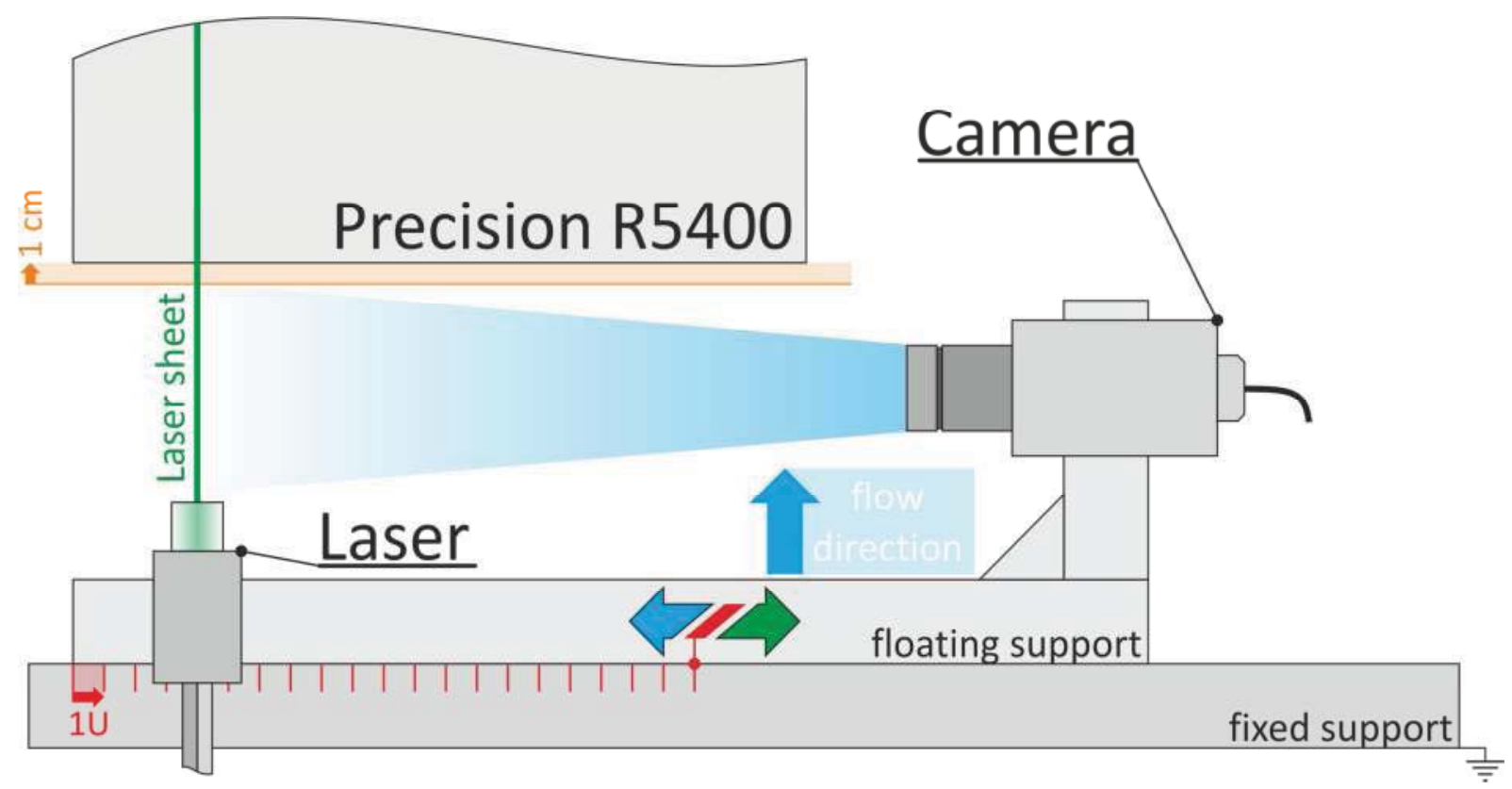

Figure 4: Experiment setup

\section{CFD model setup}

The whole geometric model of the workstation was considerably simplified. The complex internal geometry of the workstation (Figure 5) was replaced by four channels, where a pressure loss was defined for each channel (Figure 5). The pressure loss was defined in places where ventilators are located in real workstation. Thus, despite considerable geometry simplification, the position of source generating the flow within the whole setup was preserved.

The inlet areas, arising from the original geometry, were defined for particular channels (Figure 5). Due to focusing on the cabinet and workstation as a whole, the perforation of the inlet and inlet to the workstation were presented only as a rectangular inlet within the particular channels.

For defining boundary condition on channel walls, it was considered that within the inner channel walls the wall friction was not considered. Thus, despite the fact that the flow was divided by channels and there happened no mutual interaction within the channels, the flow gives the impression of compactness. By defining such boundary condition, the flow enabled to approximate the four-channel model to the one-channel solution. 


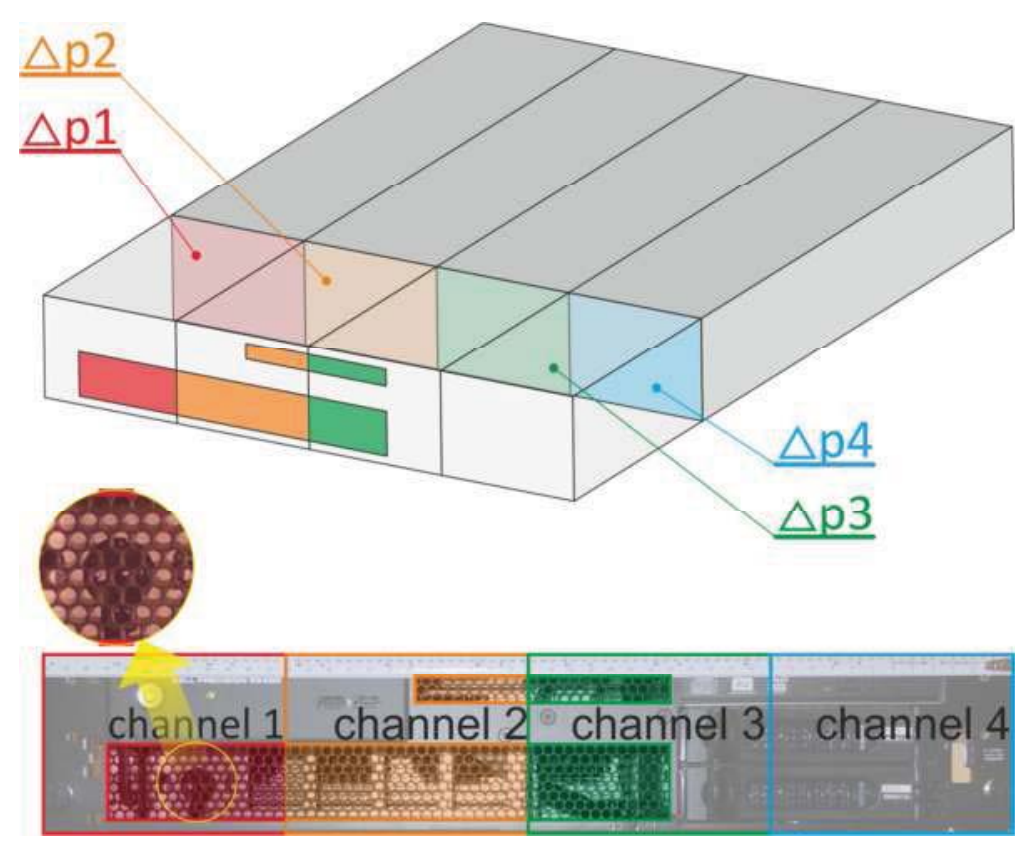

Figure 5: Four channel Workstation replacement

A simplified geometry was used for the model of the cabinet. The original geometry contained walls with thickness in order of a few millimeters. Due to the very complicated mesh creation for original geometry and due to disproportion between the wall thickness and wall width and height, all the walls in the model were replaced by a zero wall thickness. The model was hereby considerably simplified while preserving all features of original geometry and it was possible to create a complete structured non-conforming mesh.

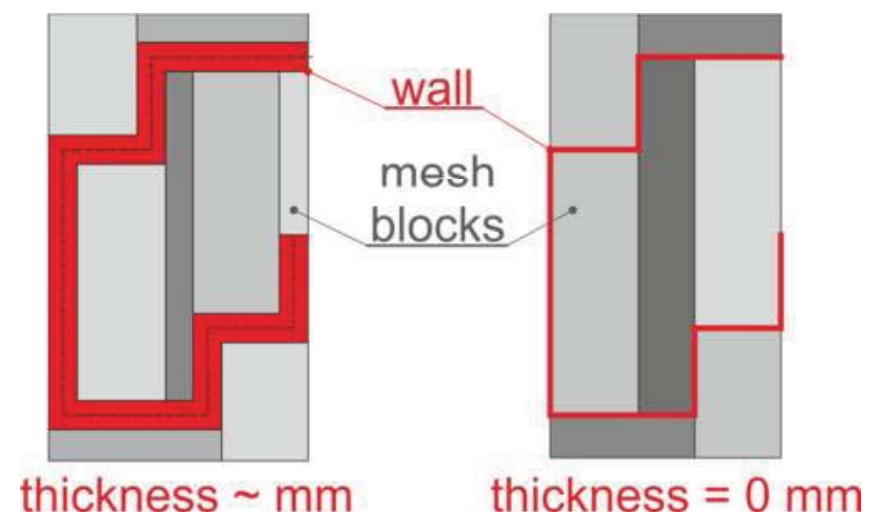

Figure 6: Wall thickness reduction

The whole volume was divided into several independent parts, which were meshed separately and the interfaces were created between resulting domains. 


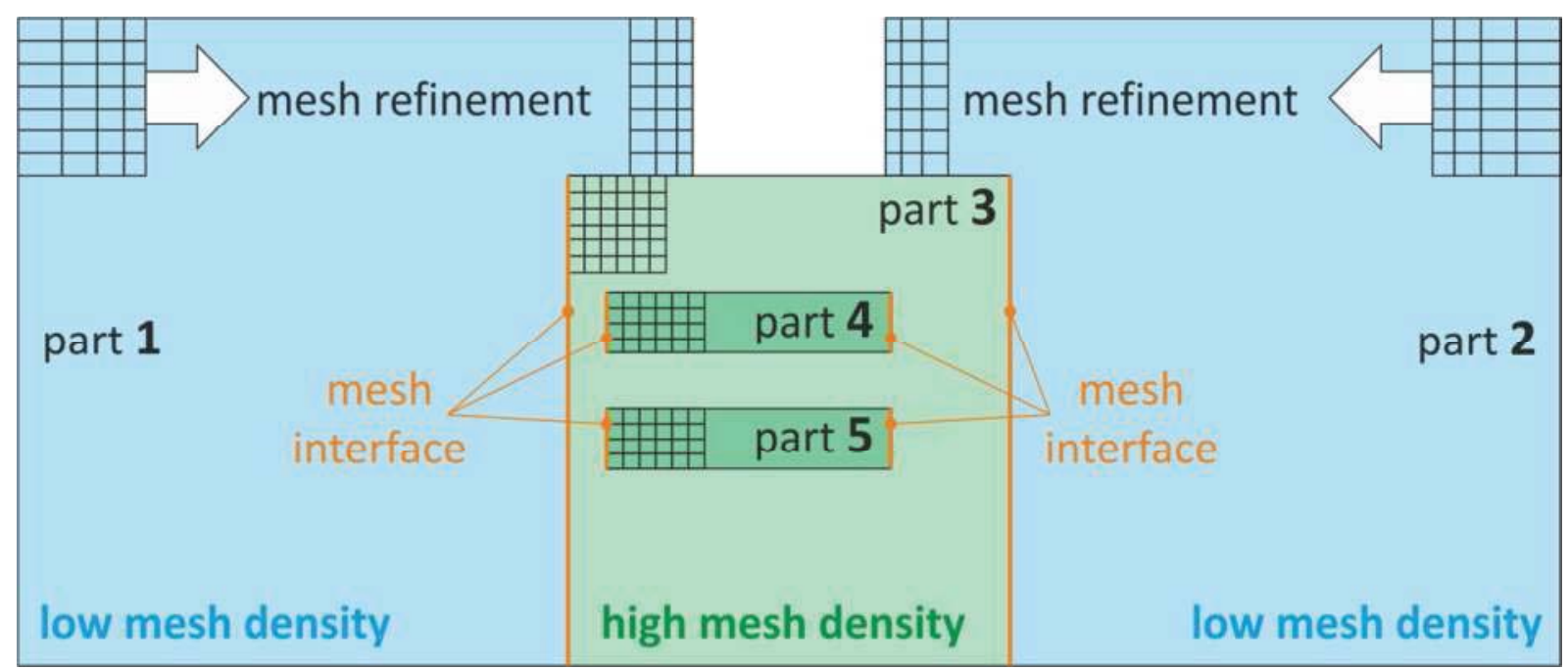

Figure 7: Computational mesh

The whole model contained 1052480 cells and was fully created with the help of hexahedron elements. For parts 1 and 2, an identical topology setting and mesh element size was used. The parts have a one plane of symmetry, but due to the asymmetry of the other parts of the compilation the symmetry condition was not used. For parts 3, 4 and 5 the identical mesh element size was used only.

A k- $\varepsilon$ model of turbulence was used for numerical solution. GGI (General Grid Interface) was used as an interface between particular meshes.

Heat transfer for the numerical model was not considered for this case.

Software Ansys 13.0 was used for mesh creation and numerical solution.

\section{Comparison of CFD and experimental data}

Four calculations were implemented in order to find suitable pressure loss in particular channels for numerical solution, where mass flow rates for particular channels were compared in the same regions from which the experimental data from the measurement were obtained. The aim was to find such a pressure loss during which the total difference of mass flow rates between the experimental measurement and numerical solution was less than $5 \%$. The pressure loss for fourth channel was not considered due to the general closing, it is mentioned in the chart below only for reasons of completeness of the whole solution (at fourth channel there are hard discs in the real workstation. The flow rate through this region is considerably limited and thus was not considered within numerical solution).

To calculate the mass flow for the numerical solution and experiment in front face of the workstation was used relationship

$$
\dot{m}=A \cdot \bar{v} \cdot \rho
$$


when speed was due to an equally large elements levels averaged in the calculation according to equation

$$
\bar{v}=\frac{\sum_{n=1}^{i} v_{i}}{n}
$$

\begin{tabular}{|c|c|c|c|c|}
\hline & \multirow{2}{*}{$\begin{array}{c}\text { Experiment } \\
\text { mass flow } \\
{[\mathrm{kg} / \mathrm{s}]}\end{array}$} & \multicolumn{3}{|c|}{ CFD \#1 } \\
\hline & & delta $p[\mathrm{~Pa}]$ & mass flow $[\mathrm{kg} / \mathrm{s}]$ & diff CFD/Exp [\%] \\
\hline Channel 1 & 0,0067 & 10 & 0,0078777 & 17,58 \\
\hline Channel 2 & 0,0078 & 10 & 0,012745 & 63,4 \\
\hline Channel 3 & 0,007 & 10 & 0,0089814 & 28,31 \\
\hline \multirow[t]{3}{*}{ Channel 4} & 0,00043976 & 0 & 0,00048615 & 10,55 \\
\hline & Experiment & \multicolumn{3}{|c|}{ CFD \#2 } \\
\hline & $\begin{array}{c}\text { mass flow } \\
{[\mathrm{kg} / \mathrm{s}]}\end{array}$ & delta $p[\mathrm{~Pa}]$ & mass flow $[\mathrm{kg} / \mathrm{s}]$ & diff CFD/Exp [\%] \\
\hline Channel 1 & 0,0067 & 10 & 0,00771 & 15,08 \\
\hline Channel 2 & 0,0078 & 5 & 0,0089882 & 15,24 \\
\hline Channel 3 & 0,007 & 7,5 & 0,0074134 & 5,91 \\
\hline \multirow[t]{3}{*}{ Channel 4} & 0,00043976 & 0 & $4,01 E-05$ & 90,88 \\
\hline & Experiment & \multicolumn{3}{|c|}{ CFD \#3 } \\
\hline & $\begin{array}{c}\text { mass flow } \\
{[\mathrm{kg} / \mathrm{s}]}\end{array}$ & delta $p[\mathrm{~Pa}]$ & mass flow [kg/s] & diff CFD/Exp [\%] \\
\hline Channel 1 & 0,0067 & 8 & 0,0069819 & 4,21 \\
\hline Channel 2 & 0,0078 & 7 & 0,010551 & 35,27 \\
\hline Channel 3 & 0,007 & 8 & 0,0076726 & 9,61 \\
\hline \multirow[t]{3}{*}{ Channel 4} & 0,00043976 & 0 & $4,27 E-05$ & 90,3 \\
\hline & Experiment & \multicolumn{3}{|c|}{ CFD \#4 } \\
\hline & $\begin{array}{c}\text { mass flow } \\
{[\mathrm{kg} / \mathrm{s}]}\end{array}$ & delta $p[\mathrm{~Pa}]$ & mass flow [kg/s] & diff CFD/Exp [\%] \\
\hline Channel 1 & 0,0067 & 8 & 0,006681 & 0,29 \\
\hline Channel 2 & 0,0078 & 4 & 0,008057 & 3,3 \\
\hline Channel 3 & 0,007 & 6,5 & 0,0066881 & 4,46 \\
\hline Channel 4 & 0,00043976 & 0 & $3,64 \mathrm{E}-05$ & 91,72 \\
\hline
\end{tabular}

Table 1: Suitable pressure jump according to mass flow rate

Color map of velocity magnitude at inlet to workstation is shown in figure 8 . Color maps are for both solutions. 


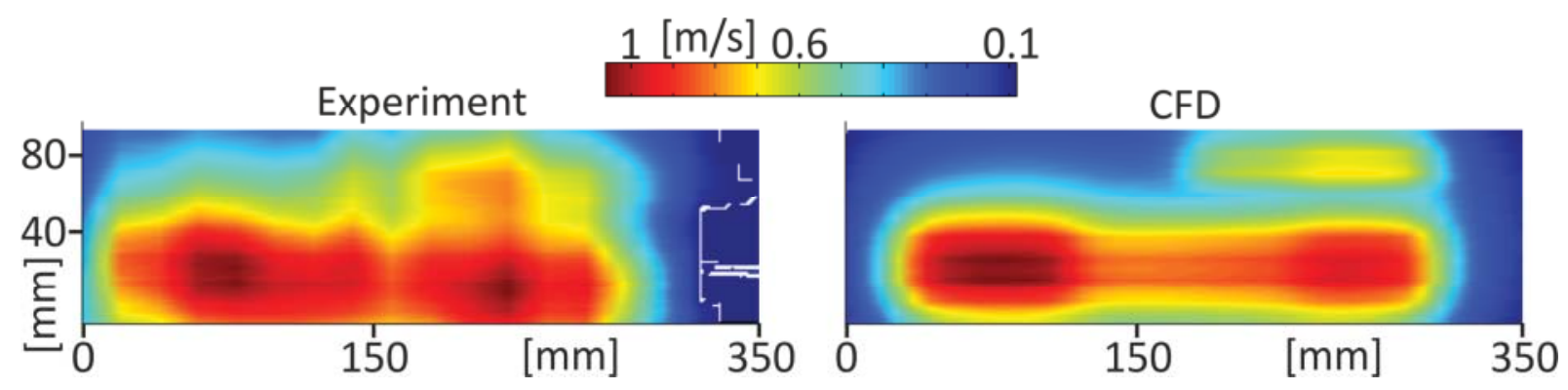

Figure 8: Velocity filed at the inlet to the workstation

In figure 9 is shown comparison between experimental and numerical data fitted to real front area of workstation.

\section{Experiment}

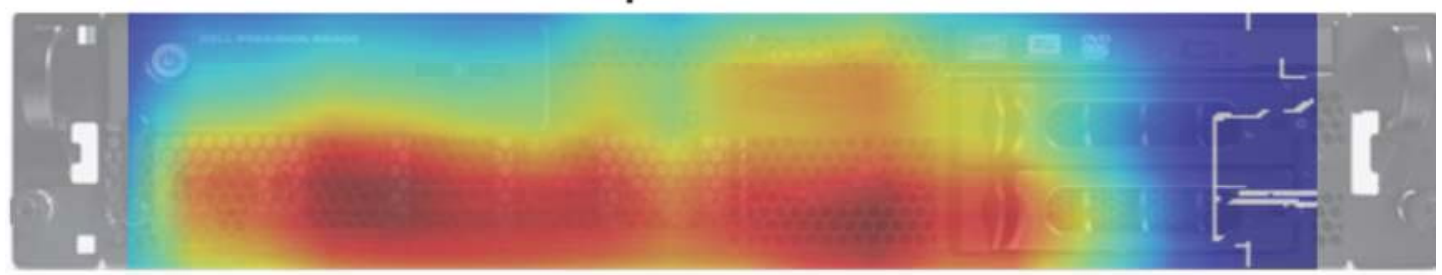

\section{CFD}
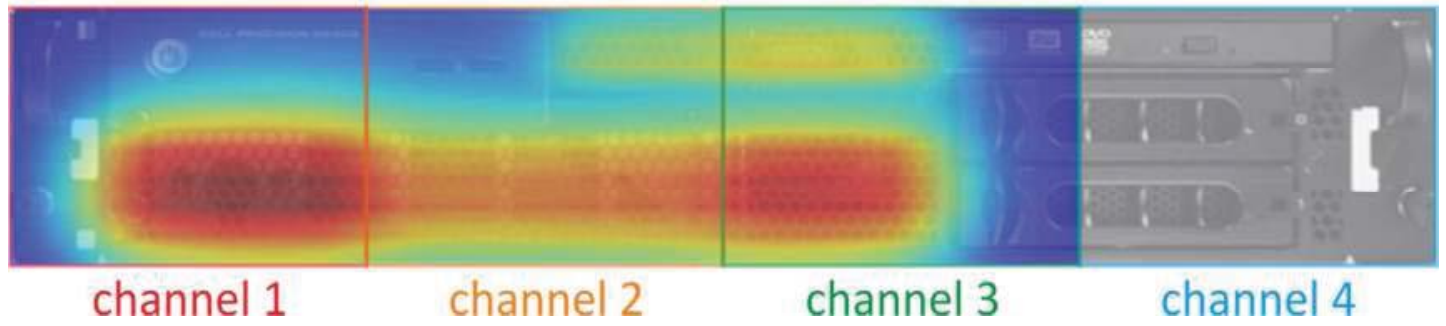

Figure 9: Location velocity field to the real geometry of the workstation

Figure 10 provides measured and computed velocity profile at inlet to workstation.

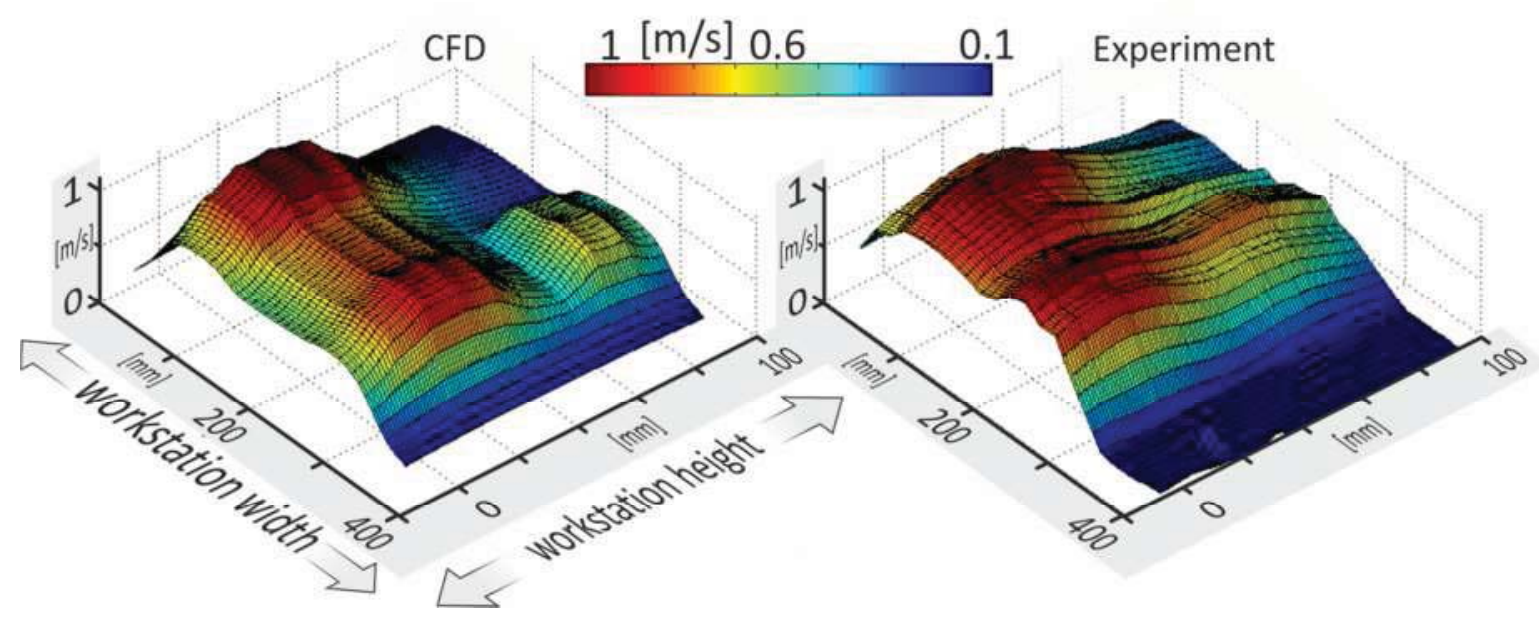

Figure 10: The shape of the velocity at the inlet to the workstation 


\section{Results}

For the first compilation, the inlet space was fully opened [FO] (Figure 2).
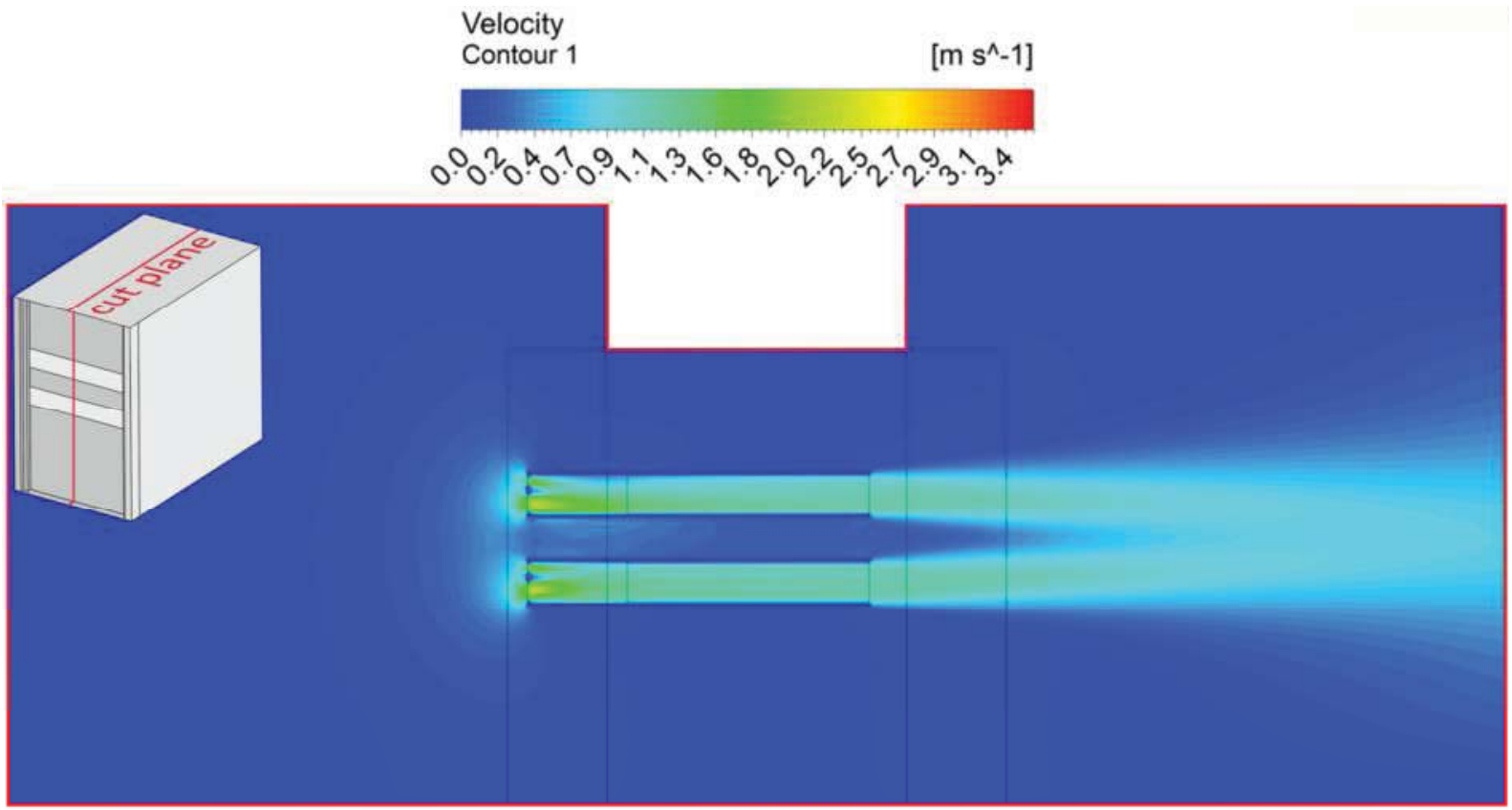

Figure 11: Contours of velocity magnitude in defined cut [FO]

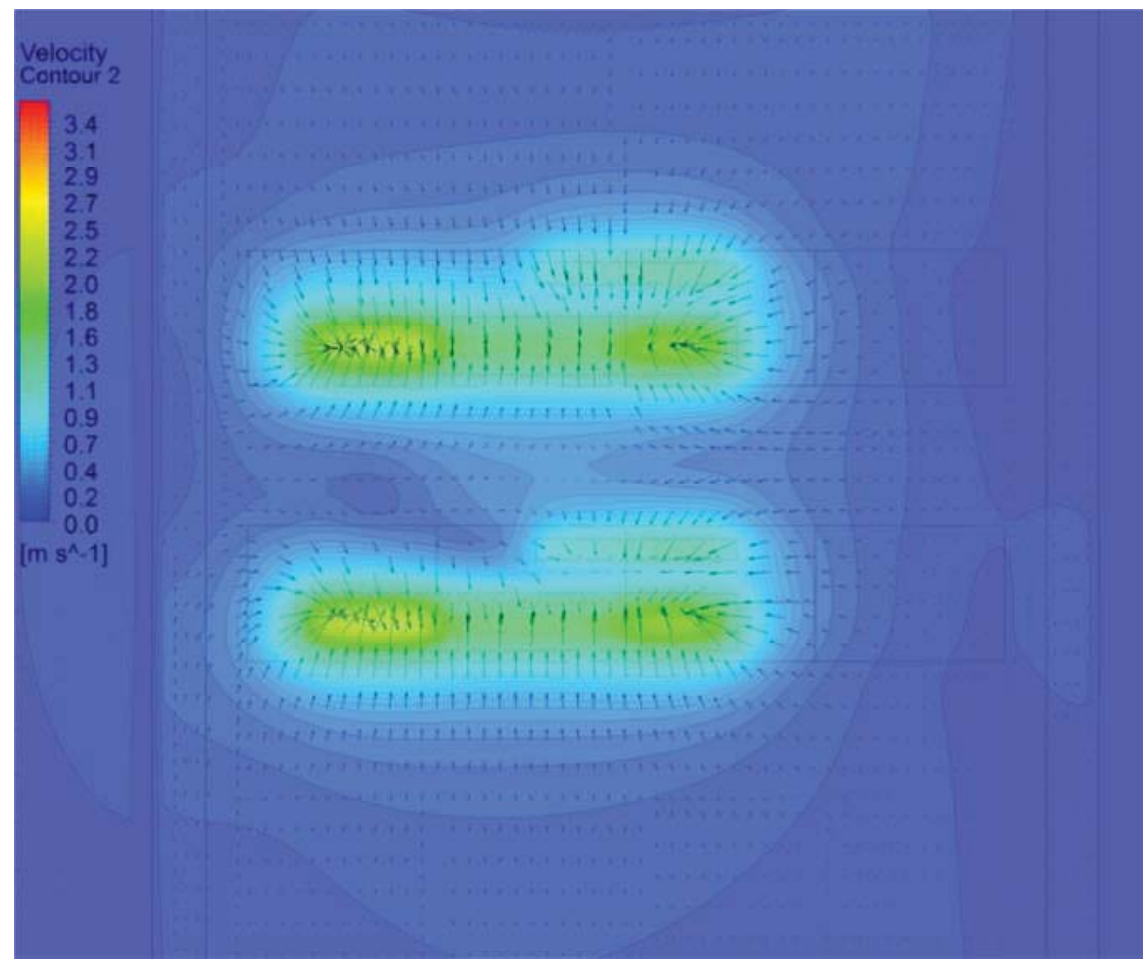

Figure 12: Contours of velocity magnitude in front face of workstations [FO] 
Figure 13 presents three cuts parallel to base where the contours of velocity are shown for whole fully opened domain.

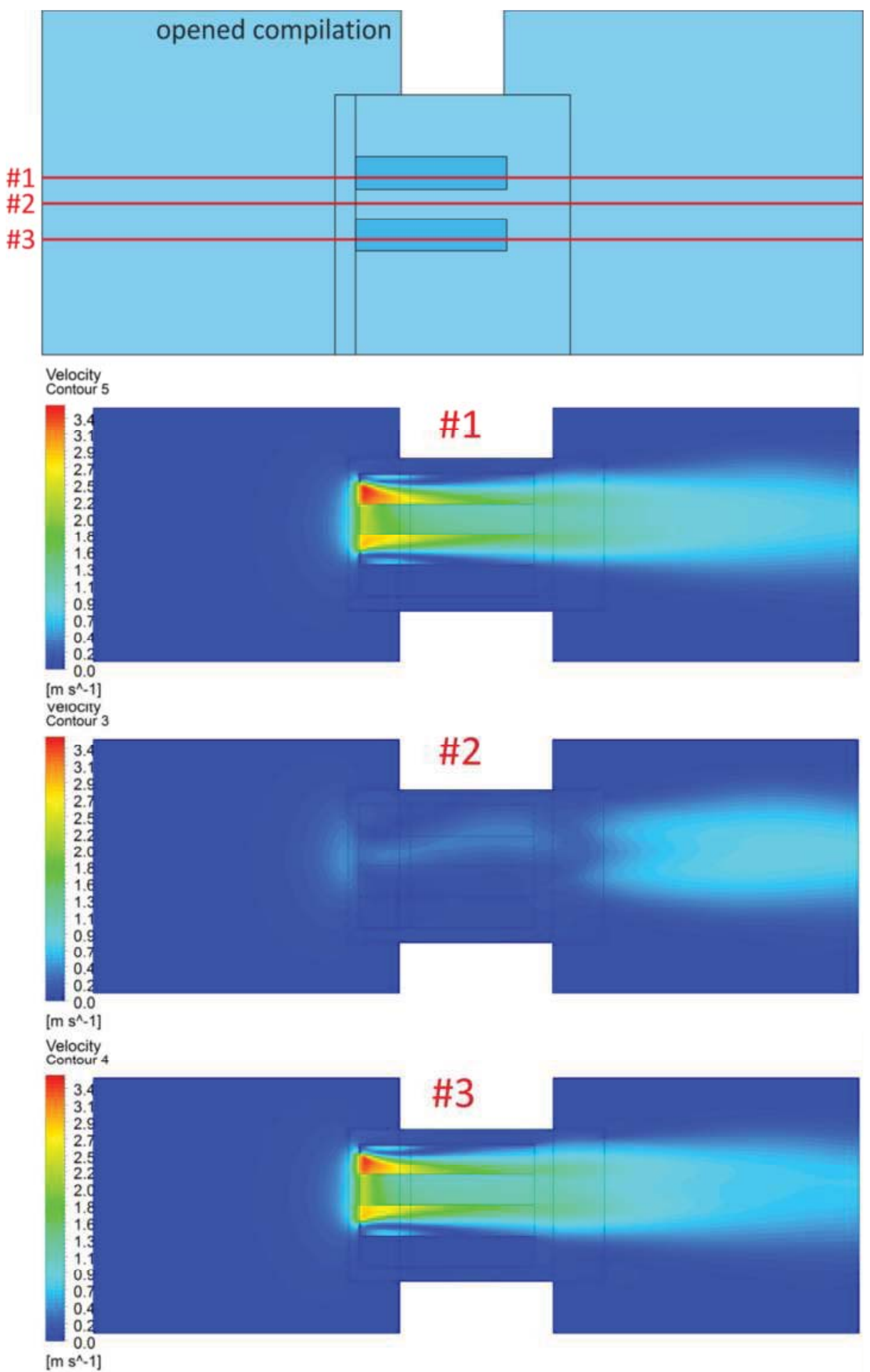

Figure 13: Contours of velocity magnitude across whole domain [FO] 


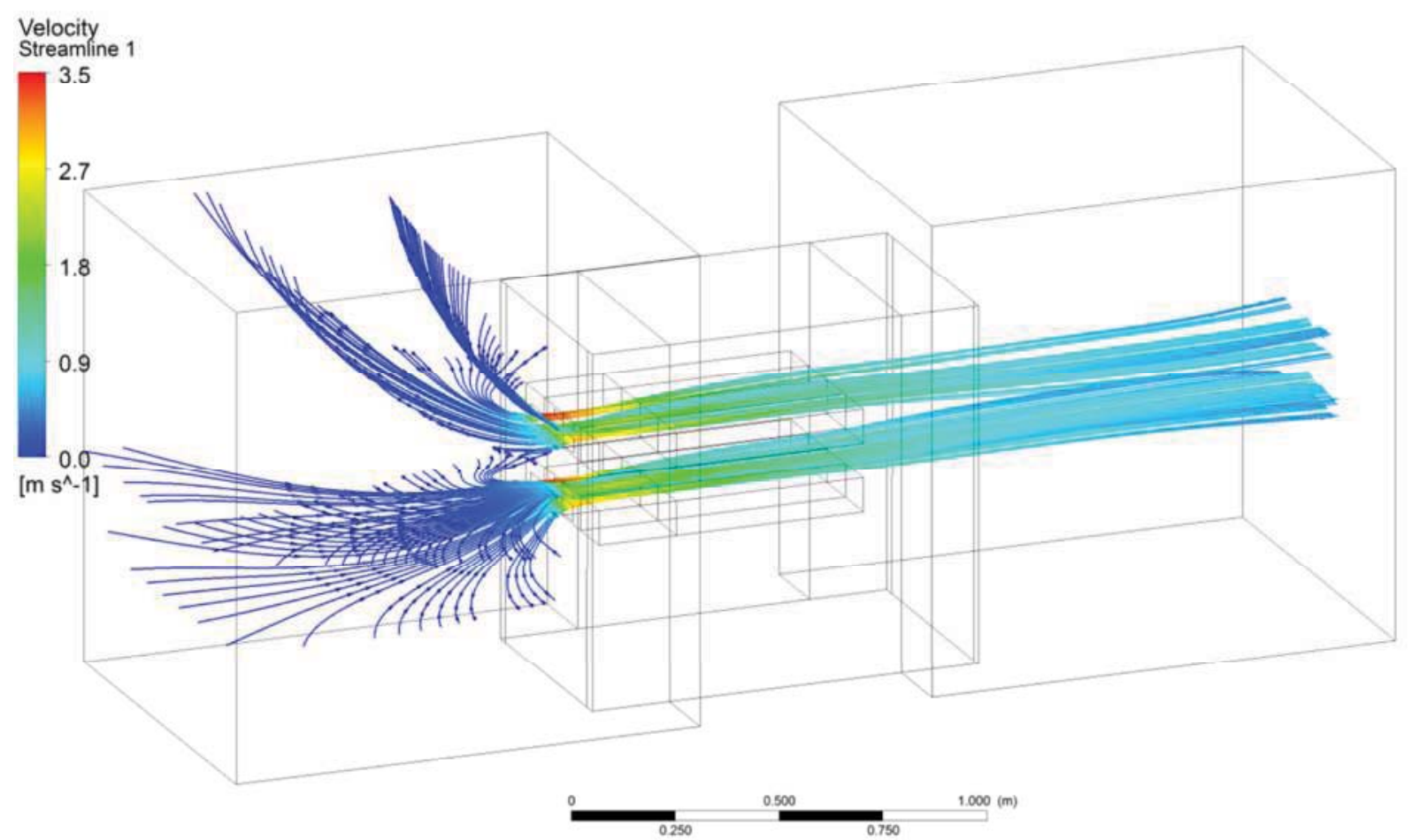

Figure 14: Velocity streamline for workstations inlet area [FO]

For the second compilation, the inlet space was fully closed [FC] (Figure 2).

Figure 15 presents three cuts parallel to base where the contours of velocity are shown for whole fully closed domain. 

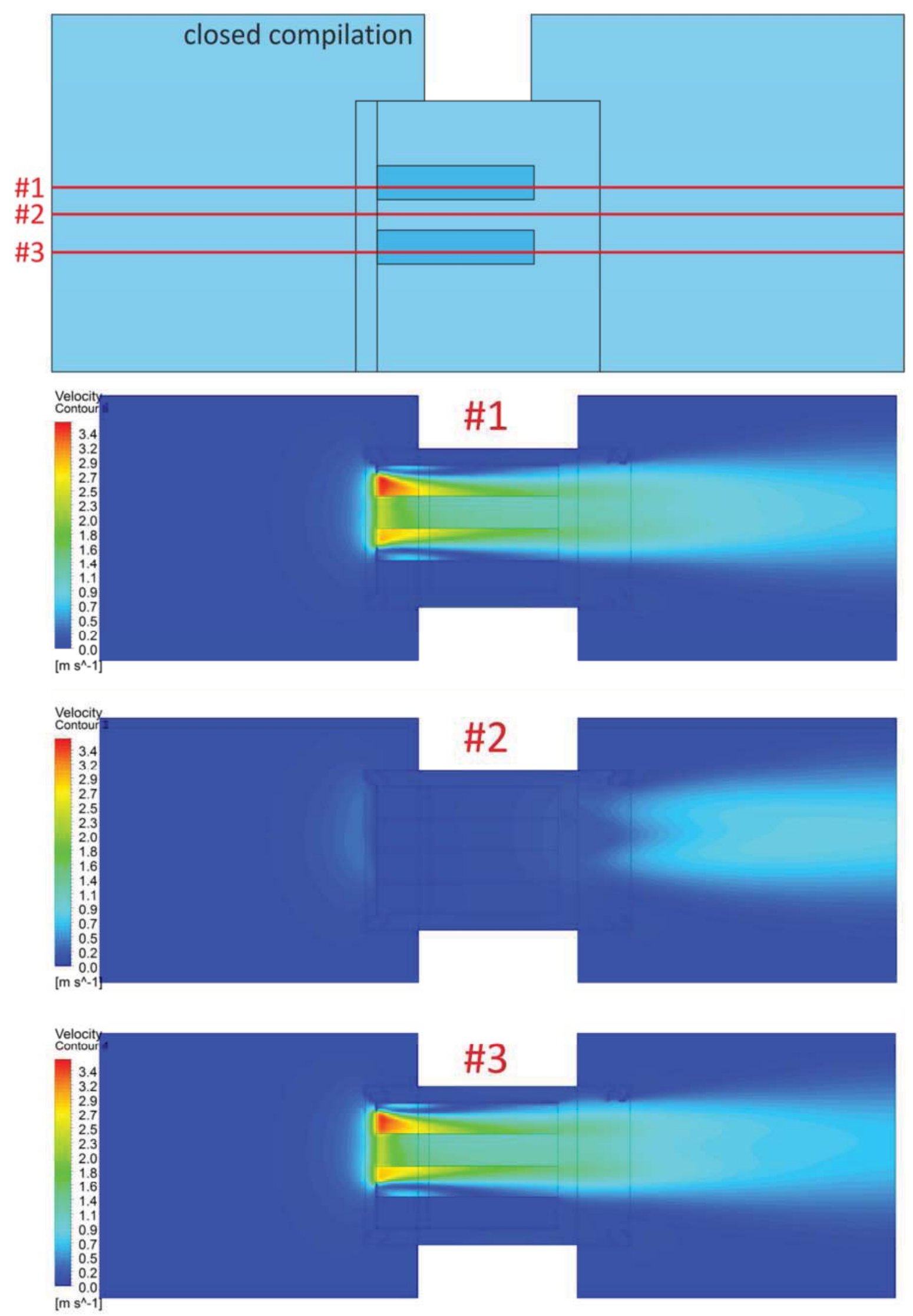

Figure 15: Contours of velocity magnitude across whole domain [FC] 


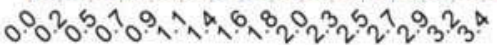

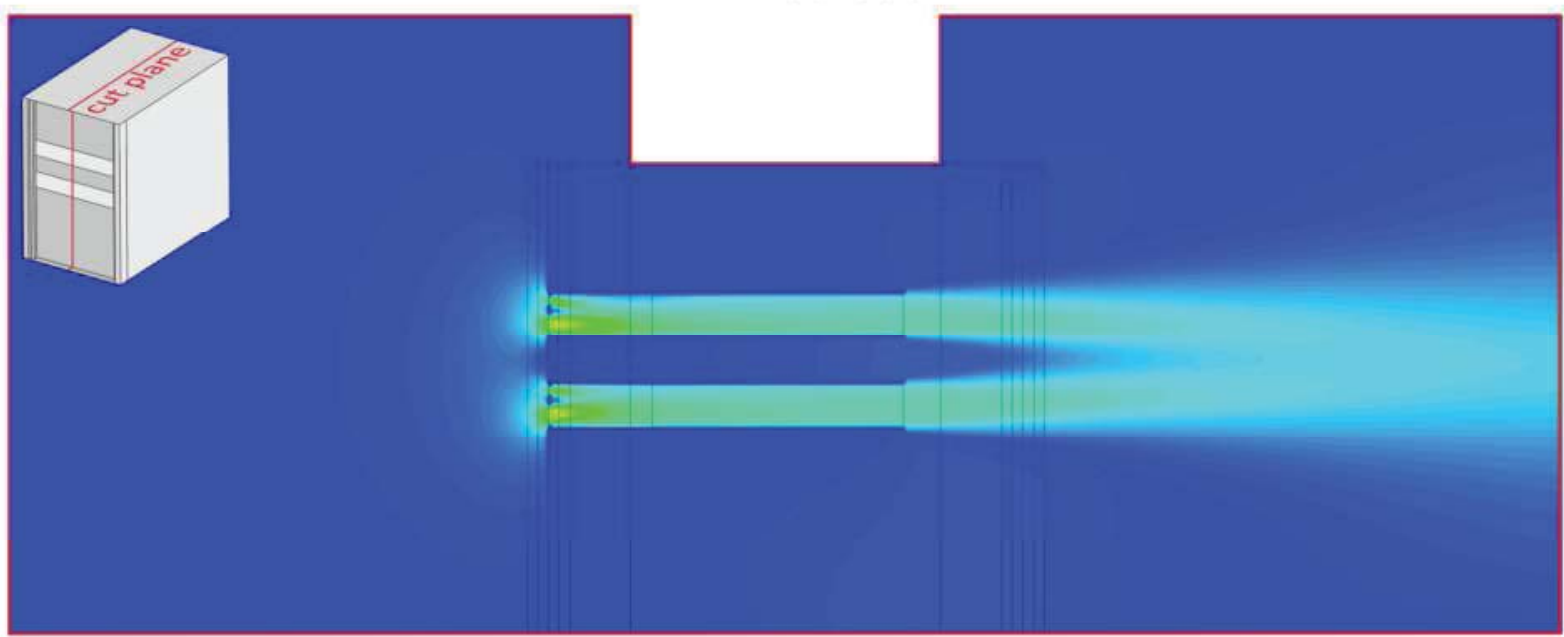

Figure 16: Contours of velocity magnitude in defined cut [FC]

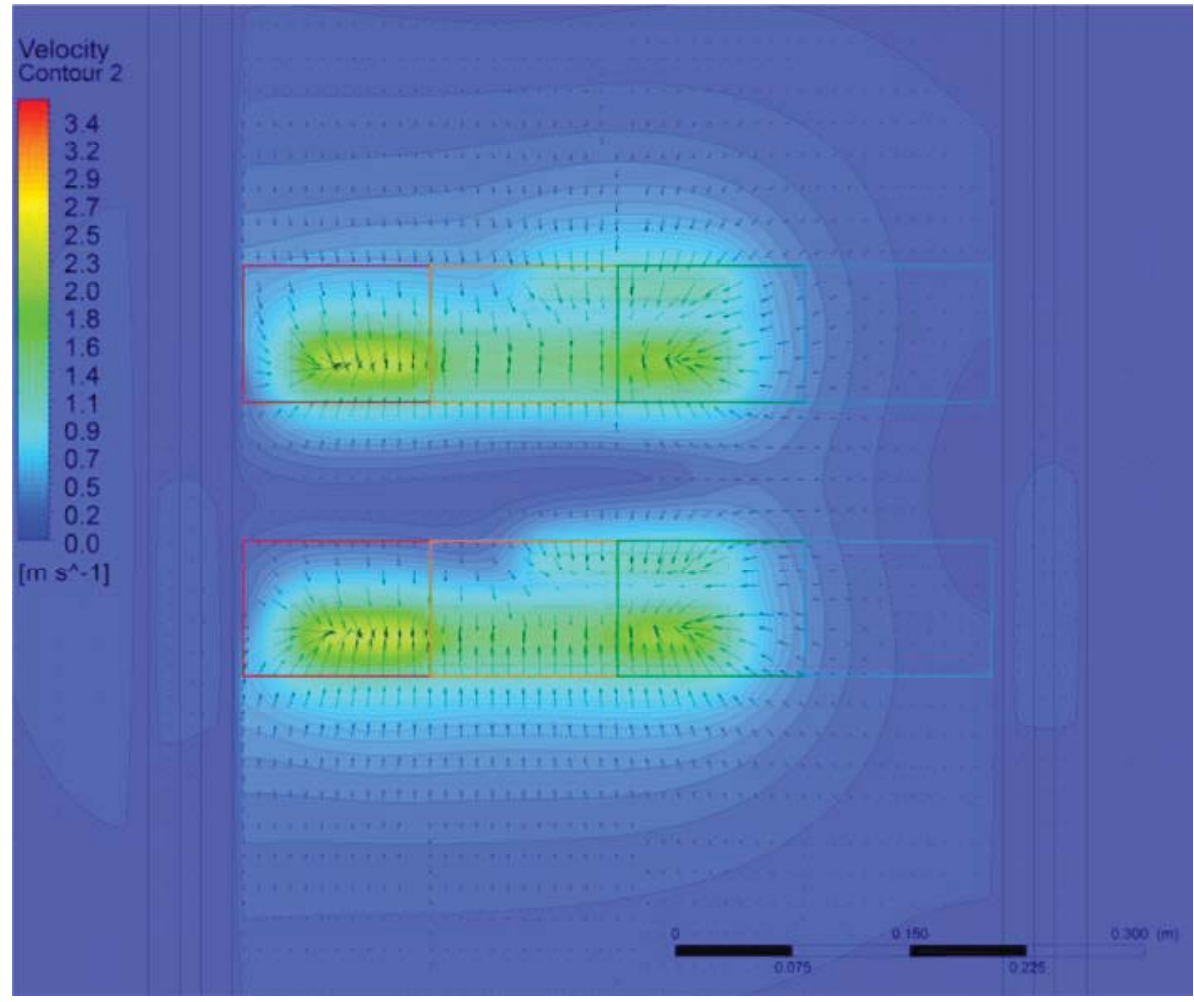

Figure 17: Contours of velocity magnitude in front face of workstations [FC] 


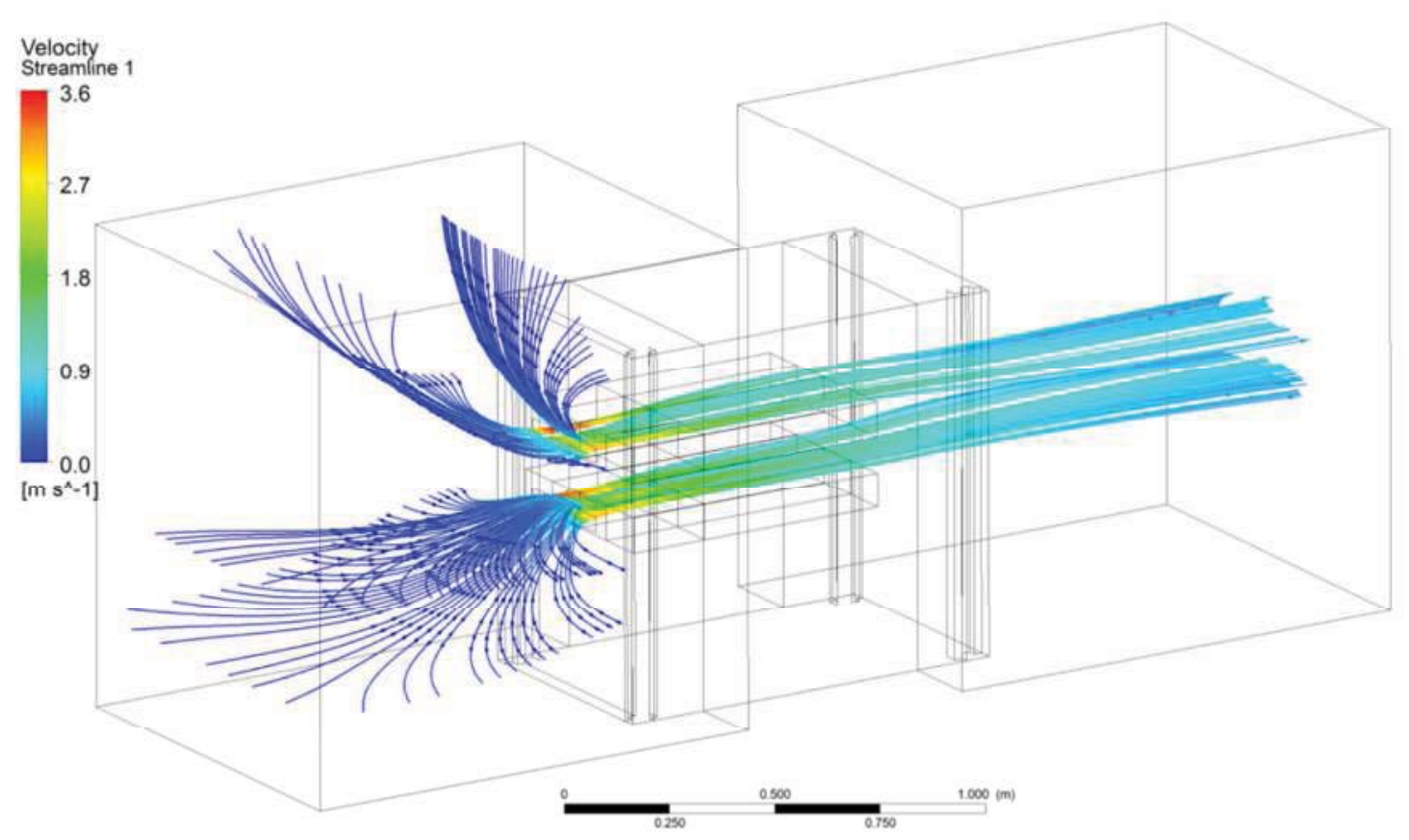

Figure 18: Velocity streamline for workstations inlet area [FC]

Difference between fully opened and fully closed compilation

In figures shown below are presents differences between fully opened and fully closed compilation. Differences are represents by absolute values of velocity contours in two cuts. In figure 19 the cut is similar to cut in figure 11 and 16. Cut in figure 20 is similar to cuts number 2 in figures 13 and 15.

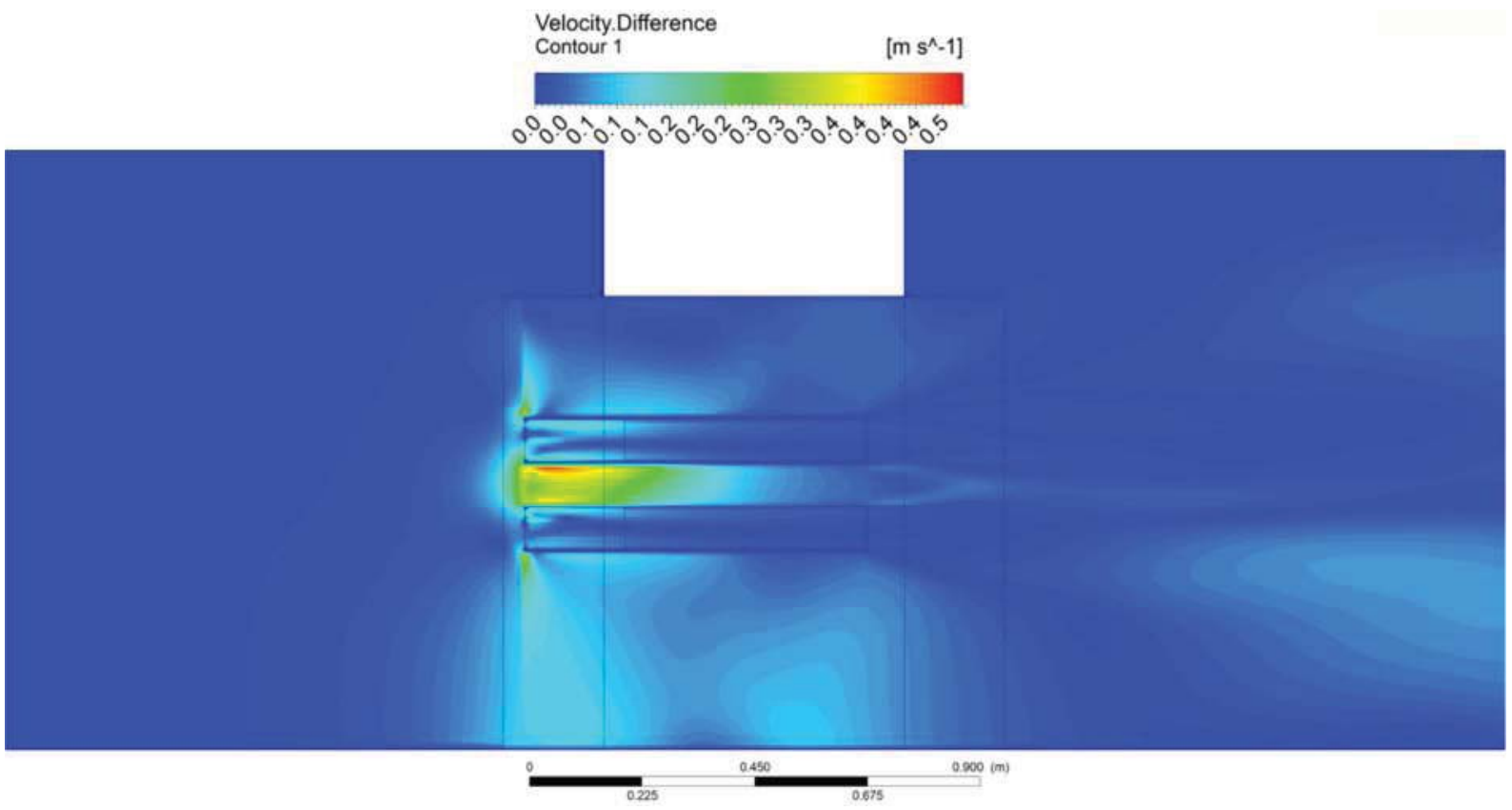

Figure 19: Velocity contours of differences between FO and FC 


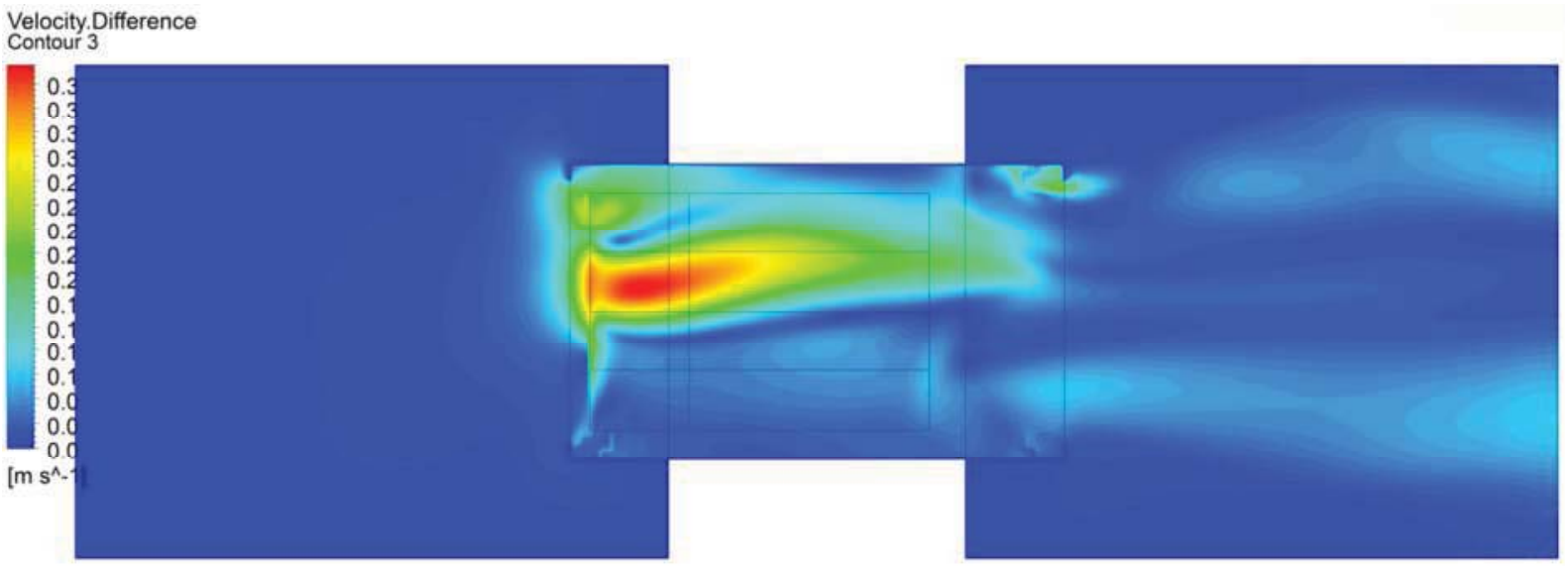

Figure 20: Velocity contours of differences between FO and FC

\section{Conclusion}

The purpose of this paper was to set up a functioning numerical model, verified by an experimental measurement, of data rack fitted with two rack workstations Dell Precision R5400. From results showed above we can say those adequate mass flow rate and pressure jumps were found for numerical solution which corresponds to experimental measurement. Differences between numerical and experimental solution were less than $5 \%$. These results will be used as a starting point for next numerical solutions where the heat transfer will be added to the model. Also another experimental measurement will be made to compare compilations mentioned in this paper with numerical results presented in this paper. Very simple channel system was presented as a replacement for very complicated geometry of real workstation. This model offer lots of ways how to improve it and find better and closer solution to the real workstation. The compilations, fully closed and fully opened were also solved and compared. As a results were printed velocity contours showing difference between these two solutions across whole domain. 